\title{
Análisis térmico de una planta de potencia de ciclo combinado utilizando enfriamiento de aire a la entrada
}

\section{Thermal analysis of a combined cycle power plant using inlet air cooling}

\author{
CASADOS-LÓPEZ, Edzel Jair†*, CRUZ-VICENCIO, Raúl, CASADOS-SÁNCHEZ, Álvaro y \\ HORST-SÁNCHEZ, Álvaro
}

Universidad Veracruzana, Facultad de Ingeniería Mecánica Eléctrica, Campus Poza Rica - Tuxpan, México.

ID 1 ${ }^{\text {er }}$ Autor: Edzel Jair, Casados-López / ORC ID: 0000-0002-0601-9242

ID $1^{\text {er }}$ Coautor: Raúl, Cruz-Vicencio / ORC ID: 0000-0002-4668-0772

ID $2^{\text {do }}$ Coautor: Álvaro, Casados-Sánchez / ORC ID: 0000-0002-3122-4571

ID $3^{\text {er }}$ Coautor: Álvaro, Horst-Sánchez / ORC ID: 0000-0002-2801-0943

DOI: 10.35429/JEA.2020.24.7.15.20

Recibido: 21 de Julio, 2020; Aceptado: 30 de Diciembre, 2020

\begin{abstract}
Resumen
En este artículo, se analiza una central eléctrica de ciclo combinado (gas-vapor), considerando enfriamiento del aire antes de entrar al compresor. En la actualidad lo que se busca son eficiencias térmicas más altas, es por lo que se ha optado por las centrales de ciclo combinado, ya que en ellas se aprovecha mejor el combustible produciendo una mayor potencia neta, todo esto ha originado modificaciones innovadoras en las centrales de ciclo combinado, mejorando el rendimiento de este. En este trabajo de investigación se toma como base una planta de ciclo combinado de $243 \mathrm{MW}$, cuya temperatura del aire al entrar al compresor es de $32^{\circ} \mathrm{C}$. Sabiendo de antemano que uno de los factores que afecta el funcionamiento de esta planta es la condición del aire al entrar al compresor, misma que al enfriarse aumentará su densidad y con ello su flujo masico obteniéndose un incremento en la potencia de la turbina de gas. En vista de esto, en este trabajo se propone qué mediante la utilización de un sistema de refrigeración mecánica, se lleve a cabo el enfriamiento del aire a $15^{\circ} \mathrm{C}$ a la entrada del compresor y con esto lograr un incremento en el rendimiento de la planta.
\end{abstract}

Planta de ciclo combinado (Gas-Vapor), Enfriamiento del aire, Compresor

\begin{abstract}
In this article, a combined cycle power station (gas-steam) is analyzed, considering air cooling before entering the compressor. Currently what is sought are higher thermal efficiencies, which is why the combined cycle power plants have been chosen, since they make better use of the fuel, producing greater net power, all of which have led to innovative modifications in the combined cycle power plants, improving the performance of this. In this research work, a $243 \mathrm{MW}$ combined cycle plant is taken as the base, whose air temperature when entering the compressor is $32{ }^{\circ} \mathrm{C}$. Knowing in advance that one of the factors that affects the operation of this plant is the condition of the air when entering the compressor, which when it cools will increase its density and with it its mass flow, obtaining an increase in the power of the gas turbine. In view of this, this work proposes that through the use of a mechanical refrigeration system, air cooling to $15^{\circ} \mathrm{C}$ is carried out at the compressor inlet and with this achieve an increase in plant performance.
\end{abstract}

Combined cycle plant (Gas-Steam), Air Cooling, Compressor

Citación: CASADOS-LÓPEZ, Edzel Jair, CRUZ-VICENCIO, Raúl, CASADOS-SÁNCHEZ, Álvaro y HORST-SÁNCHEZ, Álvaro. Análisis térmico de una planta de potencia de ciclo combinado utilizando enfriamiento de aire a la entrada. Revista de Aplicaciones de la Ingeniería. 2020. 7-24:15-20.

\footnotetext{
* Correspondencia del Autor (Correo Electrónico: edcasados@uv.mx)

$\dagger$ Investigador contribuyendo como primer autor.
} 


\section{Introducción}

$\mathrm{Al}$ aumentar la temperatura del aire a la entrada del compresor la potencia a la salida de la turbina disminuye. La reducción de la temperatura del aire a la entrada del compresor y debido a que el volumen especifico del aire es directamente proporcional a la temperatura, esto da como resultado un aumento en la densidad del aire logrando con ello una mayor tasa de flujo de masa de aire, toda vez que la tasa volumétrica es constante, por tal motivo la planta de energía mejora. Existen diferentes tipos de métodos de enfriamiento que pueden ser utilizados para enfriar el aire a la entrada del compresor, algunos de ellos son: el enfriamiento evaporativo, el de absorción y el de refrigeración mecánica entre otros. El de refrigeración mecánica es uno de los métodos más eficientes y que pueden funcionar en los climas cálidos y húmedos, este sistema no tiene limitación en la temperatura alcanzable del aire de entrada al compresor.

M.M. Alhazmy, investigó el rendimiento de las centrales eléctricas de turbinas de gas enfriando el aire de entrada al compresor. Se realizó una comparación entre dos tipos de enfriadores de aire, como el sistema de pulverización de agua y de serpentines de enfriamiento.

Se examinan las características de rendimiento para un conjunto de parámetros de diseño de operación, que incluyen la temperatura ambiente, humedad relativa, temperatura de entrada a la turbina y la relación de presiones.

Los resultados muestran que los sistemas de pulverización de agua son capaces de aumentar lo potencia y mejorar la eficiencia de la planta de energía de la turbina de gas de una manera mucho más económica que el sistema de serpentines de enfriamiento, sin embrago, opera de manera más eficiente en climas cálidos y secos. Los serpentines de enfriamiento dan un control total de las condiciones de entrada al compresor; sin embrago, consumen una cantidad considerable de energía.

Rahim K. Jassim, informa que las plantas de energía de turbinas de gas que operan en climas áridos sufren una disminución en la potencia de salida durante los calurosos meses de verano, debido a un enfriamiento insuficiente.
Un análisis de energía de un ciclo Brayton acoplado a un ciclo de refrigeración muestra una promesa para incrementar la potencia de salida. El resultado muestra que la mejora en la potencia de salida depende del grado de enfriamiento del aire al entrar al compresor.

\section{Nomenclatura}

$\mathrm{T}=$ Temperatura

$\mathrm{P}=$ Presión

$\mathrm{V}=$ Volumen

$\mathrm{\Upsilon}_{\mathrm{c}}=$ Rendimiento del compresor

$\Upsilon_{\mathrm{T}}=$ Rendimiento de la turbina

$r_{c}=$ Relación de compresión

$\dot{\mathrm{m}}=$ Flujo masico

$\mathrm{h}=$ Entalpia

he $=$ Entalpia de entrada

hs $=$ Entalpia de salida

$\mathrm{s}=$ Entropía

$\dot{\mathrm{W}}_{\mathrm{C}}=$ Trabajo del compresor

$\mathrm{W}_{\mathrm{TG}}=$ Trabajo de la turbina de gas

$\mathrm{W}_{\mathrm{TAP}}=$ Trabajo de la turbina de alta presión

$\mathrm{W}_{\mathrm{TMP}}=$ Trabajo de la turbina de media presión

$\mathrm{W}_{\mathrm{TBP}}=$ Trabajo de la turbina de baja presión

$\mathrm{W}_{\mathrm{B}}=$ Trabajo de la bomba

$\mathrm{W}_{\mathrm{n}}=$ Trabajo neto

$\mathrm{Wn}_{\mathrm{TG}}=$ Trabajo neto de la turbina de gas

$\mathrm{W}_{\mathrm{TV}}=$ Trabajo de la turbina de vapor

$\mathrm{CC}=$ Ciclo Combinado

$\mathrm{W}_{\mathrm{CC}}=$ Trabajo neto del ciclo combinado

Qs $=$ Calor suministrado 
Qr $=$ Calor rechazado

$\mathrm{Q}_{\mathrm{TG}}=$ Rendimiento térmico de la turbina de gas

$\Upsilon_{\mathrm{TV}}=$ Rendimiento térmico de la turbina de vapor

$\Upsilon_{\mathrm{CC}}=$ Rendimiento térmico del ciclo combinado

COP = Coeficiente de Operación

A continuación, se presenta el diagrama esquemático de la planta de ciclo combinado sin el sistema de refrigeración y esta consta de una turbina de gas y tres turbinas de vapor de igual capacidad.

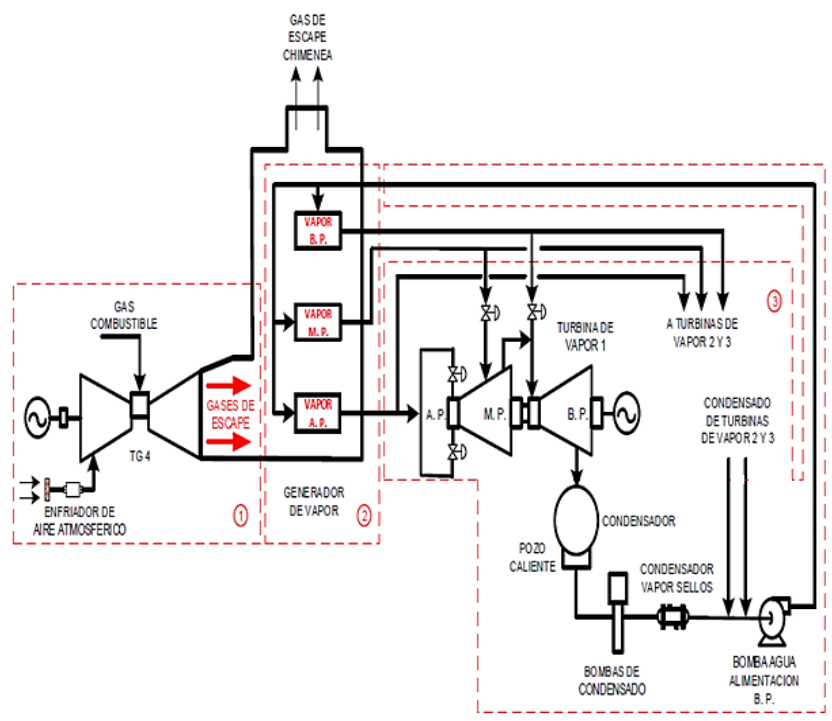

Figura 1 Diagrama esquemático de la planta de ciclo combinado, sin el sistema de refrigeración

Fuente: Elaboración propia

En seguida, se presentan los cálculos termodinámicos de las plantas de ciclo combinado:

\section{Planta del Ciclo Combinado, sin el sistema de enfriamiento del aire}

Análisis de la turbina de gas:

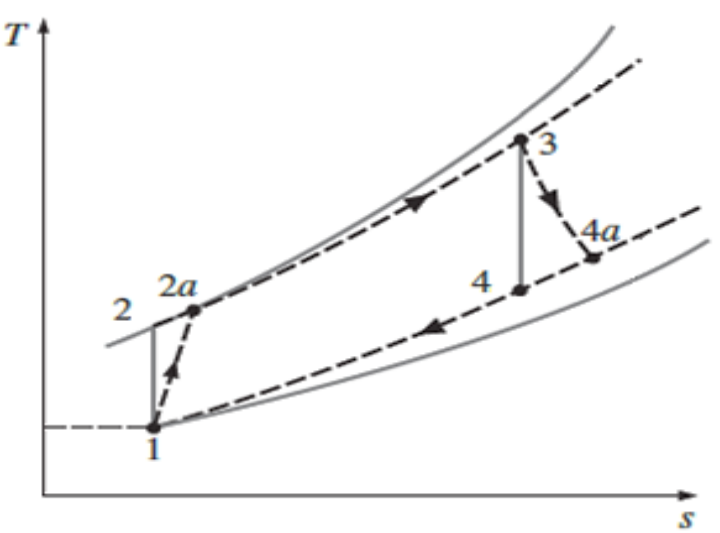

Figura 2 Diagrama T-S de la turbina de gas Fuente: Termodinámica Cengel

Datos proporcionados para el compresor y turbina de gas:

$\mathrm{T}_{1}=32{ }^{\circ} \mathrm{C}=305{ }^{\circ} \mathrm{K}$

$\Upsilon_{c}=85 \%$

$\mathrm{T}_{3}=1536^{\circ} \mathrm{K}$

$\Upsilon_{\mathrm{T}}=88.37 \%$

$\mathrm{r}_{\mathrm{c}}=14$

$\dot{\mathrm{m}}_{\text {aire }}=439.32 \mathrm{Kg} / \mathrm{seg}$

$\dot{\mathrm{m}}_{\text {combustible }}=8.56 \mathrm{Kg} / \mathrm{seg}$

De acuerdo, a los datos proporcionados para el compresor de aire y la turbina, se obtienen los siguientes valores:

\begin{tabular}{|c|c|c|c|c|}
\hline Equipo & $\mathbf{T}(\mathbf{K})$ & $\dot{\mathbf{m}}$ & \multicolumn{2}{|c|}{ Entalpia (h) } \\
\hline & entrada & & entrada & salida \\
\hline Compresor & 305 & 439.3 & 305.45 & 709.29 \\
\hline Cámara de & 696 & 447.9 & 709.29 & 1679.3 \\
\hline Turbina & 1536 & 447.9 & 1679.32 & 920.48 \\
\hline
\end{tabular}

Tabla 1 Valores de temperatura (kelvin), flujo masico $(\mathrm{Kg} / \mathrm{s})$ y entalpia $(\mathrm{KJ} / \mathrm{s})$ en cada componente de la turbina de gas

Fuente: Elaboración propia

$\dot{\mathrm{W}}_{\mathrm{c}}=\dot{\mathrm{m}}\left(\mathrm{h}_{\mathrm{s}}-\mathrm{h}_{\mathrm{e}}\right)$

$\dot{\mathrm{W}}_{\mathrm{c}}=177.414$ Mega Watts

$\mathrm{W}_{\mathrm{TG}}=\dot{\mathrm{m}}\left(\mathrm{h}_{\mathrm{e}}-\mathrm{h}_{\mathrm{s}}\right)$

$\mathrm{W}_{\mathrm{TG}}=339.869$ Mega Watts

$\mathrm{W}_{\mathrm{n}}=\mathrm{W}_{\mathrm{T}}-\mathrm{W}_{\mathrm{c}}$

$\mathrm{W}_{\mathrm{n}}=162454 \mathrm{KW}=162.45$ Mega Watts

CASADOS-LÓPEZ, Edzel Jair, CRUZ-VICENCIO, Raúl, CASADOS-SÁNCHEZ, Álvaro y HORST-SÁNCHEZ, Álvaro Análisis térmico de una planta de potencia de ciclo combinado utilizando enfriamiento de aire a la entrada. Revista de Aplicaciones de la Ingeniería. 2020 
$\mathrm{Q}_{\mathrm{s}}=\dot{\mathrm{m}}\left(\mathrm{h}_{\mathrm{s}}-\mathrm{h}_{\mathrm{e}}\right)$

$\mathrm{Qs}_{\mathrm{s}}=434.45$ Mega Watts

$\Upsilon_{\mathrm{TG}}=\mathrm{W}_{\mathrm{n}} / \mathrm{Q}_{\mathrm{s}}$

$\Upsilon_{\mathrm{TG}}=162.45 / 434.45=0.3739=37.39 \%$

Análisis de las turbinas de vapor:

En esta planta están instaladas tres turbinas de vapor de la misma capacidad por lo tanto solamente se analizará una y al final se considerarán las tres.

\begin{tabular}{|c|c|c|c|c|c|c|}
\hline \multirow[t]{2}{*}{ Equipo } & \multicolumn{2}{|c|}{ Presión } & \multirow[t]{2}{*}{ ' } & \multicolumn{2}{|c|}{ Entalpia } & \multirow[t]{2}{*}{ १.\% } \\
\hline & Ent & Sal & & Ent & Sal & \\
\hline Turbina A.P. & 7.77 & 2.14 & 24.3 & 3457.30 & 3151.25 & 77 \\
\hline Turbina M.P. & 2.1 & 1.25 & 24.3 & 3151.25 & 3030.50 & 88 \\
\hline Turbina M.P. & 1.25 & 0.46 & 26.1 & 3031.48 & 2832.56 & 88 \\
\hline Turbina B.P. & 0.46 & 0.00875 & 27.5 & 2831.68 & 2352.56 & 77 \\
\hline Bomba B.P. & 0.76 & 1.47 & 84.0 & 183.0 & 183.7 & 95 \\
\hline Bomba M.P. & 1.47 & 2.01 & 5.61 & 183.7 & 673.7 & 95 \\
\hline Bomba A.P. & 2.01 & 9.87 & 75.2 & 673.7 & 678.5 & 95 \\
\hline
\end{tabular}

Tabla 2 Valores de presión (Mega pascales), flujo masico $(\mathrm{kg} / \mathrm{s})$ y entalpia $(\mathrm{KJ} / \mathrm{s})$ en cada componente de la turbina de vapor

Fuente: Elaboración propia

Rendimiento térmico del ciclo de vapor:

$\mathrm{W}_{\mathrm{T}}=\left(\mathrm{W}_{\mathrm{TAP}}+\mathrm{W}_{\mathrm{TMP}}+\mathrm{W}_{\mathrm{TMP}}+\mathrm{W}_{\mathrm{TBP}}\right) \bigcap_{\mathrm{mec}}$

$\mathrm{W}_{\mathrm{T}}=(28.745)(0.9586)=27.55$ Mega Watts

$\mathrm{W}_{\mathrm{B}}=\left(\mathrm{W}_{\mathrm{BBP}}+\mathrm{W}_{\mathrm{BMP}}+\mathrm{W}_{\mathrm{BAP}}\right) \bigcap_{\mathrm{mec}}$

$\mathrm{W}_{\mathrm{B}}=3005.28 \mathrm{KW}$

$\mathrm{W}_{\mathrm{B}}=3005.28 \mathrm{KW} / 3=1001.76 \mathrm{KW}$

$\mathrm{W}_{\mathrm{B}}=1.0017$ Mega Watts

$\mathrm{W}_{\mathrm{n}}=\mathrm{W}_{\mathrm{TV}}-\mathrm{W}_{\mathrm{B}}$

$\mathrm{W}_{\mathrm{n}}=26.549$ Mega Watts

El trabajo neto por las tres turbinas seria:

$\mathrm{W}_{\mathrm{n}}=26.549 \times 3=79.647$ Mega Watts

$\mathrm{Q}_{\mathrm{s}}=\dot{\mathrm{m}}\left(\mathrm{h}_{\mathrm{e}}-\mathrm{h}_{\mathrm{s}}\right)=249.043$ Mega Watts

$\mathrm{Q}_{\mathrm{TV}}=\mathrm{W}_{\mathrm{n}} / \mathrm{Qs}_{\mathrm{s}}$

$\Upsilon_{\mathrm{TV}}=79647 / 249043=0.3198=31.98 \%$

El trabajo neto del ciclo combinado es:

$\mathrm{W}_{\mathrm{CC}}=\mathrm{W}_{\mathrm{TG}}+\mathrm{W}_{\mathrm{TV}}=\mathbf{2 4 2 . 0 9}$ Mega Watts combinado es:

El Rendimiento térmico del ciclo

$\chi_{\mathrm{CC}}=\chi_{\mathrm{TG}}+\chi_{\mathrm{TV}}-\chi_{\mathrm{TG}} \mathrm{\chi}_{\mathrm{TV}}$

$\Omega_{\text {CC }}=0.3739+0.3198-0.3739(0.3198)$

$\mathrm{\Upsilon}_{\mathrm{CC}}=0.5742=57.42 \%$

A continuación, se muestra el diagrama de la misma planta, pero ahora considerando el sistema de enfriamiento del aire.

Planta de ciclo combinado, considerando el sistema de enfriamiento del aire

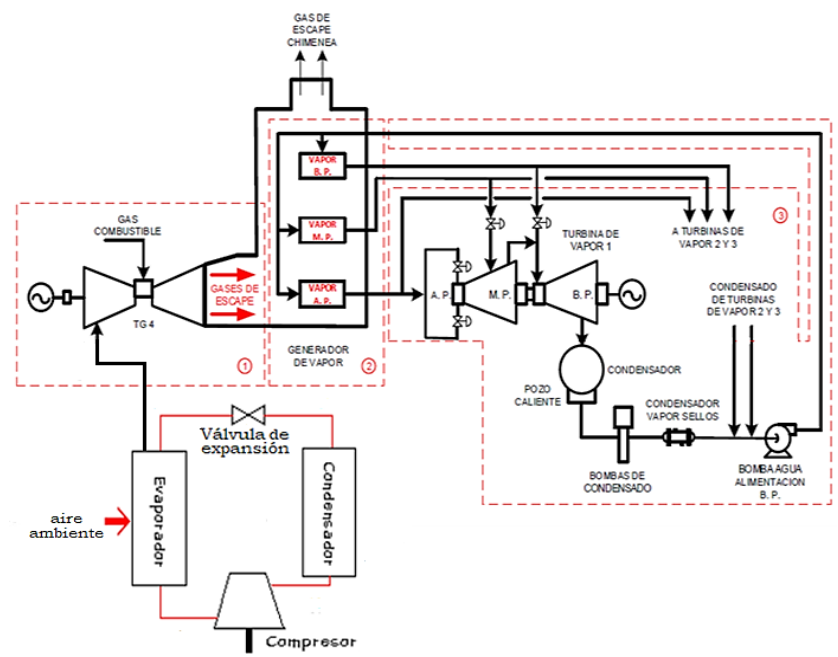

Figura 3 Diagrama esquemático de la planta de ciclo combinado con ciclo de refrigeración por compresión de vapor

Fuente: Elaboración propia

Ciclo de refrigeración por compresión de vapor

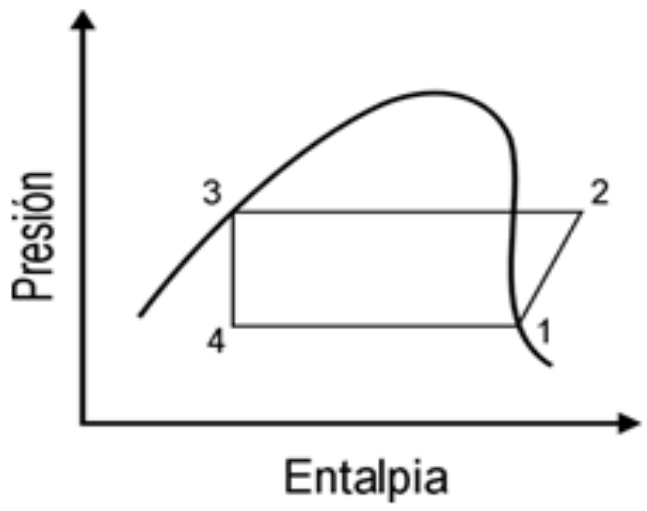

Figura 4 Diagrama presión entalpia del ciclo de refrigeración

Fuente: Termodinámica Cengel 


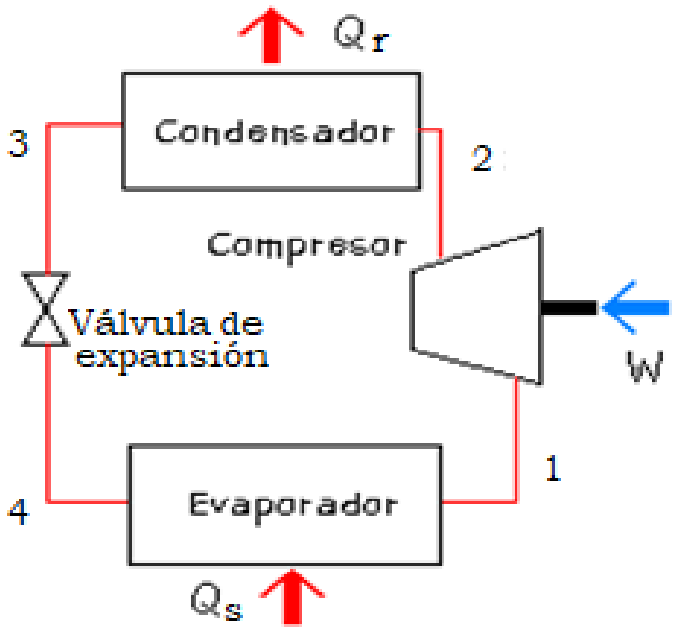

Figura 5 Diagrama esquemático del ciclo de refrigeración por compresión de vapor

Fuente: Elaboración propia

Se puede realizar un análisis del ciclo simple de un sistema estándar de refrigeración por compresión de vapor suponiendo:

- Un proceso de flujo constante a través de cada componente

- Variación de energía cinética y potencial despreciable

- No hay transferencia de calor ni caída de presión en conexiones y tuberías.

$\mathrm{W}_{\mathrm{C}}=\mathrm{h}_{2}-\mathrm{h}_{1}$

$\mathrm{COP}=\left(\mathrm{h}_{1}-\mathrm{h}_{4}\right) /\left(\mathrm{h}_{2}-\mathrm{h}_{1}\right)$

Se realizan los cálculos térmicos de cada una de las componentes de la turbina de gas considerando que el aire, que entra al compresor tiene una temperatura de $15^{\circ} \mathrm{C}$.

\begin{tabular}{|l|c|c|c|c|}
\hline \multicolumn{1}{|c|}{ Equipo } & \multirow{2}{*}{ T } & $\dot{\mathbf{m}}$ & \multicolumn{2}{|c|}{ Entalpia (h) } \\
\cline { 4 - 5 } & entrada & & entrada & salida \\
\hline Compresor & 288 & 463.8 & 288.38 & 670.18 \\
\hline $\begin{array}{l}\text { Cámara de } \\
\text { combustión }\end{array}$ & 659.5 & 472.4 & 670.18 & 1679.3 \\
\hline Turbina & 1536 & 472.6 & 1679.32 & 920.48 \\
\hline
\end{tabular}

Tabla 3 Valores de temperatura (Kelvin), flujo masico $(\mathrm{Kg} / \mathrm{s})$ y entalpia $(\mathrm{KJ} / \mathrm{s})$ en cada componente de la turbina de gas considerando una temperatura más baja del aire.

Fuente: Elaboración Propia

Análisis de la turbina de gas con enfriamiento del aire:
$\dot{\mathrm{W}}_{\mathrm{c}}=\dot{\mathrm{m}}\left(\mathrm{h}_{\mathrm{s}}-\mathrm{h}_{\mathrm{e}}\right)=177.090$ Mega Watts

$\mathrm{W}_{\mathrm{T}}=\dot{\mathrm{m}}\left(\mathrm{h}_{\mathrm{e}}-\mathrm{h}_{\mathrm{s}}\right)=358.454$ Mega Watts

$\mathrm{W}_{\mathrm{n}}=181.360$ Mega Watts

$\mathrm{Q}_{\mathrm{s}}=\dot{\mathrm{m}}\left(\mathrm{h}_{\mathrm{s}}-\mathrm{h}_{\mathrm{e}}\right)=476.70$ Mega Watts

$\Upsilon_{\mathrm{TG}}=81.36 / 476.70=0.3804=38.04 \%$

Debido a que el trabajo y rendimiento térmico en la turbina de vapor es el mismo, en este caso ya no se volverá a calcular, pero se dan los resultados de los trabajos netos.

Trabajo neto de la turbina de Vapor:

$\mathrm{W}_{\mathrm{n}}=79.647$ Mega Watts

El trabajo neto del ciclo combinado con sistema de enfriamiento del aire es:

$\mathrm{W}_{\mathrm{CC}}=\mathrm{W}_{\mathrm{TG}}+\mathrm{W}_{\mathrm{TV}}=261$ Mega Watts

El rendimiento térmico del ciclo combinado con sistema de enfriamiento del aire quedaría:

$\chi_{\mathrm{CC}}=\chi_{\mathrm{TG}}+\chi_{\mathrm{TV}}-\chi_{\mathrm{TG}} \chi_{\mathrm{TV}}$

$\Omega_{\mathrm{CC}}=0.3804+0.3198-0.3804(0.3198)$

$\mathrm{\Upsilon}_{\mathrm{CC}}=0.5786=57.86 \%$

\section{Conclusiones y Resultados}

Después de analizar esta investigación, puede concluirse que en una planta de potencia de ciclo combinado (Gas-Vapor) cuando el aire tiene diferentes temperaturas al entrar en el compresor se obtiene lo siguiente:

Cuando el aire a la entrada del compresor tiene una temperatura de $32{ }^{\circ} \mathrm{C}$, se obtuvieron los siguientes resultados:

$\mathrm{Wn}_{\mathrm{TG}}=162.45$ Mega Watts

$\mathrm{W}_{\mathrm{CC}}=242.09$ Mega Watts

$\Upsilon_{\mathrm{CC}}=57.42 \%$

Considerando que se analizará el mismo ciclo combinado, pero ahora tomando en cuenta que el aire al entrar al compresor de la turbina, pasa por un sistema de refrigeración por compresión de vapor y entra a una temperatura de $15{ }^{\circ} \mathrm{C}$, se obtuvieron los siguientes resultados: 
$\mathrm{Wn}_{\mathrm{TG}}=181.36$ Mega Watts

$\mathrm{W}_{\mathrm{CC}}=261.0$ Mega Watts

$\Upsilon_{\mathrm{CC}}=57.86 \%$

Estos resultados nos indican que introduciendo el aire al compresor con una temperatura de $15{ }^{\circ} \mathrm{C}$ se incrementa la potencia de la turbina de gas en $11.64 \%$ y el rendimiento térmico del ciclo combinado se incrementa muy poco, faltando por analizar los costos económicos por la instalación del sistema de enfriamiento del aire para analizar la conveniencia de este.

\section{Referencias}

Alhazmy, M.M., Najjar, Y.S.H, (2004). Augmentation of gas turbine performance using aire coolers, Applied termal Engineering 24.

Cerri, G., (2015). "Parametric Analysis of Combined Gas Steam Cycles", ASME Paper No. 82-GT-95.

Fraize, W. E., and Kinney, C., (1979). Effects of Steam Injection on the Performance of Gas Turbine Power Cycles. "ASME Journal of Engineering for Power, Vol. 101, pp. 217-227.

Hernández-Fernández, N. J., Zumalacárregui-de Cárdenas, L., \& Perez-Ones, O. (2020). Simulación de condiciones de operación y fluidos de trabajo para ciclos Rankine orgánicos. Revista de investigación, Desarrollo e Innovación, 10(2).

Jassim, R. K., Zaki, G.M., Alhazmy, M.M., (2010). Thermo - Economic analysis of a gas turbine power plant with cooled air intake, Yanbu Journal of Engineering ans Sciencie.

Kakaras, E, Doukelis, A., Prelipceanu, A., Karellas, S., (2006). Inlet air cooling Methods for gas turbine based power plants, Journal of Engineering for GasTurbines and Power, Vol. 128,312-317.

Kehlhofer R (1997). Combined cycle Gas and steam Turbine power Plant pennwell publishing company ISBN-0-87814-736-5.
L. J. Nayak, D. Mahto. (2014). Parametric Analysis of Combined Cycle Power Plant Using Inlet Vapour Compression Cycle. International Journal of Emerging Technology and Advanced Engineering.

Ondryas, I.S., Wilson, D. A., Kawamoto, M., Haub G.L., (1991). Option in gas Turbine power augmentation using inlet air chilling, engineering gas turbine and power, ASME 113 203-2012

Pfenninger, H., (1982). "Combined steam and gas turbine power stations, Brown Boveri, Paper No. CH-T 040013E

Quiñones Gutierrez, E. M., \& Saldaña Castillo, E. A. (2020). Diseño de una central termoeléctrica de cogeneración de $5 \mathrm{MW}$ para la planta Backus-Motupe.

Sanjay (2013), Energy and exergy analysis of combined cycle systems with different bottoming cycle configurations, Int J.Energy Res. 37: 899912.

Sanjay, Onkar Singh, B N Prasad, (2008). Infuence of different means of Turbine blade cooling on thermodynamic performance of combined cycle, Applied thermal Engineering 28 2315-2326.

Santos, A.P., (2012). Analysis of gas Turbine Performance with inlet air Cooling techniques, appliedtoBraziliansites, J. Aerospace. Technol Manag. Vol. 4. No. 3, pp.314-353.

Sonneuschain, H., (1982). A Modular Optimizing Calculation Method of Power Station Energy Balance and Plant Efficiency. "ASME Journal of Engineering of Power, Vol. 104, pp. $225-259$

Nag P.K. (2009) Power Plant Engineering. Tata McGraw-Hill Publishingcompany limited. New Delhi ISBN-13: 978-0-07-064815- 9.

Yadav R (2004) steam and gas turbine and Power plant Engineering. Central publishing House, Allahabad. 